\title{
Demography and Short Term Functional Outcome of Soft Tissue Sarcoma Treated with Limb Salvage Surgery
}

\section{Suresh Pandey'}

'Department of Orthopaedics, College of Medical Sciences, Bharatpur, Chitwan, Nepal.

\begin{abstract}
Introduction

Limb salvage surgery with or without radiotherapy and/or chemotherapy is the mainstay of treatment of Soft Tissue Sarcoma (STS). Though, many studies are found regarding the demographics and result of such malignancy from other parts of the world, such data is not available from our country. This study aimed to analyze the demographic data and functional outcome of STS treated with limb salvage surgery.
\end{abstract}

\section{Methods}

It is a retrospective review of 16 cases of soft tissue sarcoma of extremities and trunk managed with limb salvage surgery followed by adjuvant radiotherapy and/or chemotherapy in indicated cases from 2012 to 2017. Demographic data and functional score after 2 years of surgery were analysed and presented.

\section{Results}

There were 14 male and 2 female cases of STS with mean age of 32.88 years. The most common STS was liposarcoma (37.5\%) followed by rhabdomyosarcoma (25\%) and synovial sarcoma (18.8\%). Extremity and lower limb were the most common sites. Mean MSTS functional score at the last follow up was 24.56 (81.83\%) with range from 19-29 (63.33\%-96.66\%). There were good to excellent results in 13 cases (81.25\%) and moderate result in 3 cases (18.75\%) according to MSTS functional score. There was positive correlation of MSTS with age, radiotherapy, chemotherapy and negative correlation with grade of tumor.

\section{Conclusions}

This study showed good to excellent functional results in $81.25 \%$ of cases as per Musculoskeletal Tumor Society Score (MSTS) with positive correlation with radiotherapy, chemotherapy, age and negative correlation with grade of tumor.

Keywords: soft tissue sarcoma; functional outcome; wide local excision; adjuvant radiotherapy.

Correspondance: Dr. Suresh Pandey, Department of Orthopaedics, College of Medical Sciences, Bharatpur-10, Chitwan, Nepal. Email: drsuresh.orthonepal@gmail.com. Phone: +977-9845047228. 


\section{INTRODUCTION}

Soft tissue sarcoma (STS) are relatively rare, heterogenous group of malignant tumor and constitute $<1 \%$ of all malignancy. ${ }^{1}$ Extremities are the most common site for STS (60\%) followed by trunk and other regions. ${ }^{2}$ Because of its complexity and uncommon presentation, many orthopedic surgeons have limited experience in dealing with these malignancy. Treatment of these tumors are complex issue and involves multidisplinary approach with active role of operating surgeon, pathologist, oncoradiation specialist, oncophysician, nurses and family members. Mainstay of treatment for STS is wide local excision with tumor free margin. Postoperative adjuvant Radiotherapy (RT) can be beneficial in high grade tumor or positive tumor margin. Role of Chemotherapy (CT) is debatable as these malignancy are least chemoresponsive and sensitivity varies according to tumor types. Limb salvage surgery is the treatment of choice in most of the cases unlike amputation in the past. ${ }^{3,4}$ Combination of tumor free surgical resection of tumor at amenable sites with postoperative adjuvant radiotherapy in indicated cases have resulted in acceptable functional results and complication rate. ${ }^{5-7}$ Prognosis is not up to the expectation despite treatment especially for high grade tumors. About half of them present with metastatic state during follow up. Approximately half of all patients with intermediate or highgrade soft tissue sarcomas develop metastatic disease and the overall survival is approximately $50 \%$ at 5 years. ${ }^{8}$

There are different ways to analyze the outcome of tumor surgery such as functional outcome, oncological outcome and quality of life after the treatment. Functional outcome is assessed by different measures and Musculoskeletal Tumor Society Score (MSTS) is one of the commonest and widely practiced method. Studies are lacking from our country about the demographics and functional outcome of limb salvage surgery of STS. Present study aimed to analyze the demographic data and functional outcome of
STS after limb salvage surgery with or without adjuvant radiotherapy or chemotherapy.

\section{METHODS}

This is a retrospective review of soft tissue sarcoma cases managed with limb salvage surgery between January 2012 to January 2017 by multidisciplinary team approach in tertiary care centre. Approval for the study was taken from the institutional ethical committee.

Inclusion criteria were clinicoradiologically and cytologically or histopathologically proven new cases of soft tissue sarcoma or recurrence without encasement of major neurovascular bundle in extremities and trunk.

Exclusion criteria were cases not amenable for limb salvage surgery such as encasement of major neurovascular bundle or huge or fungating mass not amenable for safe surgical excision and distant metastatic state at the time of presentation.

Details of demography, diagnosis and management were obtained from case charts. Diagnosis was based on clinical history, examination and investigation findings. All the imaging investigations including MRI were done before invasive procedures. Extent of lesion, size, status of major neurovascular structures, bones and joints were assessed to decide the limb salvage surgery. FNAC was done in all the cases for cytological confirmation and incision biopsy was done where diagnosis was doubtful or could not be established with needle cytology. Details of disease nature, treatment plan, operative excision, chance of residual limb function, postoperative neurological complications, infection, chance of local recurrence and metastasis were explained to patient and caretaker beforehand. Consent was taken for the operation accordingly. Possible need of postoperative adjuvant radiotherapy and chemotherapy were explained in advance.

Operation was done under suitable anesthesia following standard principles of sarcoma surgery 
taking care of obtaining tumor free margin. Caution was taken at the same time to balance the postoperative function by preservation of major nerves and vessels. Haemostasis was secured and wound was closed with negative suction drain after wide local excision of the malignant mass. Physiotherapy for ROM exercise, muscle strengthening exercise were started as per the pain tolerance from $2^{\text {nd }}$ postoperative day. Postoperative course and outcome records were noted from discharge summary and OPD card about wound status, infection, ROM, function of the limb, neurovascular deficit, evidence of local recurrence or pulmonary metastasis and questionnaires for MSTS score was completed at least 2 years after operation. High grade tumor or tumor with positive margin were advised for adjuvant RT after 3 weeks of operation. Adjuvant chemotherapy was added in high grade STS and Rhabdomyosarcoma by the oncophysician.

MSTS score consists of six items and are completed together by a physician and patient. Pain, function, and emotional acceptance are measured for both upper and lower extremities. For the lower extremity, use of walking aids, gait, and walking are evaluated. Hand positioning, dexterity, and lifting ability are evaluated for the upper extremity. All six categories are rated on a scale from zero to five, with five representing normal function. Function is expressed as percentage of the maximum score. ${ }^{9}$ The MSTS score is intended to measure patients' functional outcome and impairment. Excellent result was defined as $75 \%$ to $100 \%$, good as $70 \%$ to $74 \%$, moderate as $60 \%$ to $69 \%$, fair as $50 \%$ to $59 \%$, and poor as $<50 \%$.

Data were entered into Excel chart and statistical calculation was done using SPSS software (SPSS 21, SPSS Inc., Chicago, IL, USA). Frequency and mean were calculated using descriptive statistics and MSTS score was calculated to grade the functional outcome at least 2 years after the operation. Correlation between MSTS score with age, sex, grade, tumor size, postoperative radiotherapy and chemotherapy was done with Pearson's correlation test. $P$ value of $<0.05$ was considered as statistical significance level.

\section{RESULTS}

\begin{tabular}{|c|c|c|c|c|c|l|c|}
\hline \multicolumn{7}{|l|}{ Table 1. Demographic data and clinical characteristics of soft tissue sarcoma. $n=$ nerve. } \\
\hline Age & Sex & $\begin{array}{c}\text { Tumor } \\
\text { size (cm) }\end{array}$ & stage & Grade & Site & Diagnosis & $\begin{array}{c}\text { Duration of } \\
\text { symptoms (mth) }\end{array}$ \\
\hline 12 & M & 21.5 & III & 2 & leg & Rhabdomyosarcoma & 9 \\
\hline 10 & M & 17 & III & 3 & gluteal & Rhabdomyosarcoma & 7 \\
\hline 54 & M & 28 & I & 1 & thigh & Liposarcoma & 9 \\
\hline 34 & F & 18 & III & 3 & leg & Synovial sarcoma & 5 \\
\hline 52 & M & 22 & I & 1 & axilla & synovial sarcoma & 12 \\
\hline 41 & M & 30 & I & 1 & thigh & Liposarcoma & 10 \\
\hline 16 & M & 8 & II & 1 & forearm & malignant $n$ sheath & 4 \\
\hline 12 & M & 24 & III & 2 & leg & Rhabdomyosarcoma & 7 \\
\hline 10 & M & 18 & III & 2 & gluteal & Rhabdomyosarcoma & 5 \\
\hline 54 & M & 28 & I & 1 & thigh & Liposarcoma & 9 \\
\hline 34 & F & 16 & III & 3 & leg & Synovial sarcoma & 5 \\
\hline 52 & M & 26 & I & 1 & axilla & Liposarcoma & 12 \\
\hline 41 & M & 18 & I & 1 & thigh & Liposarcoma & 6 \\
\hline 16 & M & 6 & II & 1 & forearm & Malignant $n$ sheath & 4 \\
\hline 52 & M & 18 & I & 1 & axilla & Liposarcoma & 12 \\
\hline 36 & M & 14 & I & 1 & leg & Fibrosarcoma & 12 \\
\hline
\end{tabular}


There were total of 16 cases of soft tissue sarcoma managed with limb salvage surgery with mean age of 32.88 years (range 10-54). There were male predominance of $87.5 \%(n=14)$ and female $12.5 \%(\mathrm{n}=2)$. The mean duration of symptoms was 8.0 months (range 4-12) and mean follow up duration was 30.19 months (range 24-45). The most common site of sarcoma was leg $(31.3 \%)$ followed by thigh $(25 \%)$, axilla $(18.8 \%)$, and gluteal and forearm region $(12.5 \%)$ each. Lower extremity was the most common site of STS $(56.31 \%)$. The most common types of STS was liposarcoma (37.5\%) followed by rhabdomyosarcoma (25\%), synovial sarcoma $(18.8 \%)$, malignant nerve sheath tumor $(12.5 \%)$ and fibrosarcoma (6.3\%). Most of the STS were stage I (50\%) followed by stage III (37.5\%) and
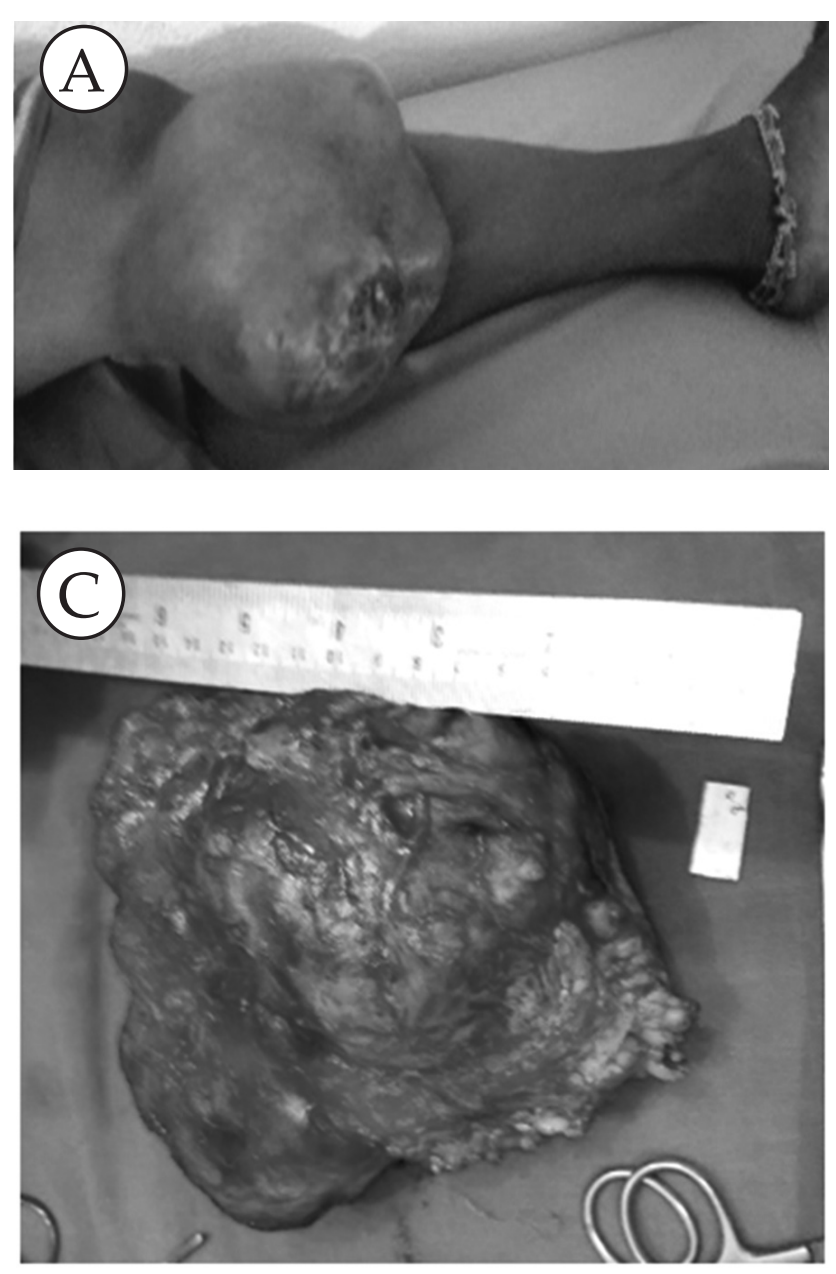

to be $62.5 \%, 18.8 \%, 18.8 \%$ for grade 1,2 and 3 respectively. Demographic summary of the STS have been shown in the table 1.

Mean diameter of tumor (as measured by maximum diameter of the tumor after excision) was $19.53 \mathrm{~cm}$ (range 6-30). All the STS were treated with wide local excision following the principle of sarcoma surgery followed by adjuvant RT in 8 STS (50\%) where there was positive margin and/or high grade tumor and postoperative adjuvant chemotherapy in 4 cases of STS $(25 \%)$ to those with high grade tumor. No case received preoperative neoadjuvant RT or CT. There was no cases of resection of major neurovascular structures or reconstruction, bone resection or reconstruction of soft tissue with
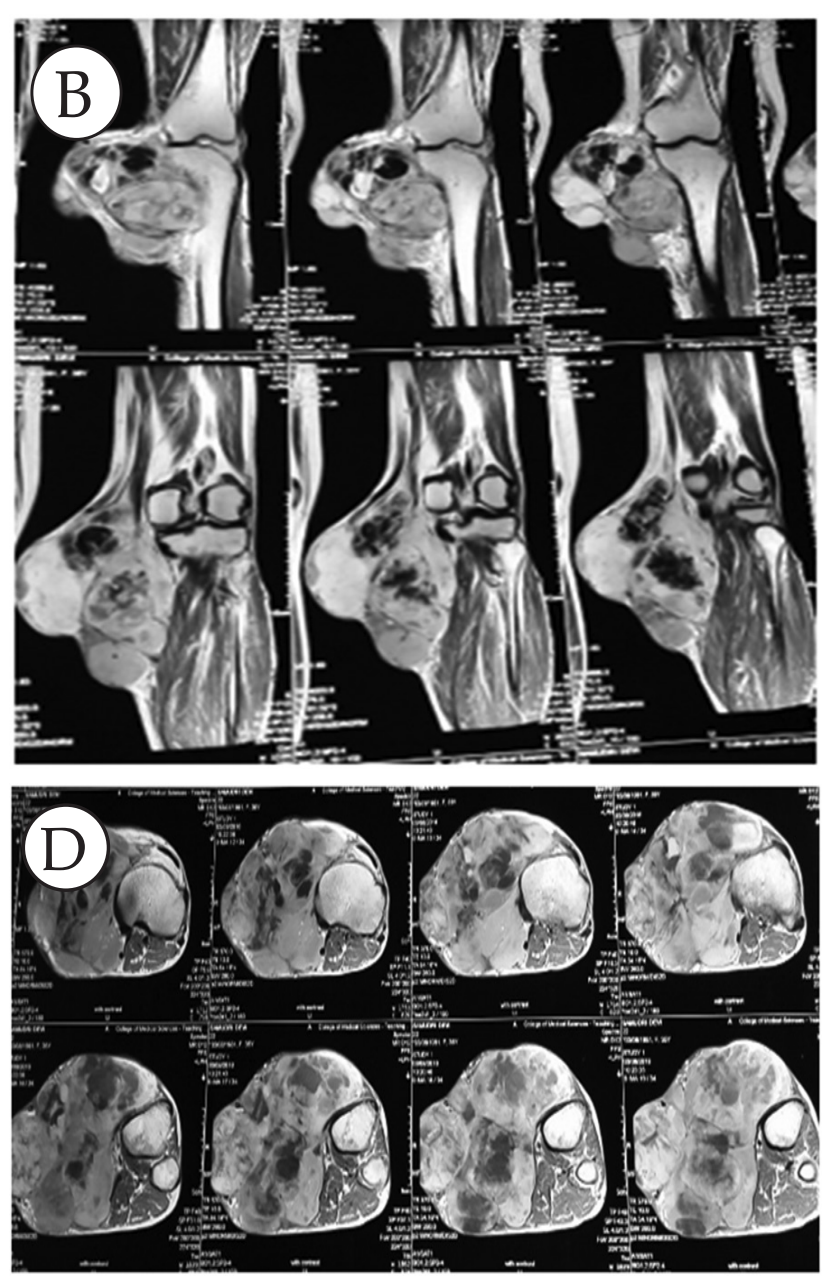

Figure 1. A, Large soft tissue mass of sarcoma with extension to knee and leg; B and C, coronal and axial section MRI showing heterogenous, multilobulated soft tissue mass with sparing of bone and joint suggestive of synovial sarcoma; D synovial sarcoma mass after wide local excision. 
local recurrence in a case of rhabdomyosarcoma at 30 months of follow up with evidence of lung metastasis in the same case. There was no mortality till minimum of 2 years of follow up. There was no wound related complications in any of them. One case of STS of gluteal region had sciatic nerve palsy and one case of malignant nerve sheath tumor of forearm had posterior interosseous nerve palsy postoperatively which improved to motor power of grade 3 at 6 months.

Mean MSTS functional score at the last follow up was $24.56(81.83 \%)$ with range from 19-29 (63.33\%-96.66\%). There were good to excellent results in 13 cases $(81.25 \%)$ and moderate result in 3 cases(18.75\%) according to MSTS functional score.

There was negative correlation between tumor stage and grade with final MSTS score with significance level $<0.05$ on Pearson coefficient test whereas there was positive correlation between age, adjuvant chemotherapy and postoperative RT with final MSTS score on bivariate analysis. Association between tumor size and MSTS score was not statistically significant.

\section{DISCUSSION}

Soft tissue sarcoma arises from varying group of tissue such as fat, muscle, nerve cells, vessels and result of limb salvage treatment has improved in recent decades because of better surgical techniques and understanding, multidisciplinary approach and adjuvant therapy. ${ }^{10,11}$ Functional results after the treatment of STS depends upon multiple parameters such as age, tumor grade, staging, tumor margin, bone resection, plastic or microvascular surgical procedures, postoperative radiotherapy, chemotherapy and tumor size. Current study showed positive correlation between age, RT and chemotherapy on final functional MSTS score and significant negative correlation between stage and grade of tumor with MSTS score.
Amputation used to be the mainstay of treatment of sarcoma before 1980 but limb salvage surgery has been established as rule in recent 2 decades. ${ }^{10}$ Wide local excision has increased risk of local recurrence due to risk of leaving residual tumor while amputation has increased morbidity and psychological distress. Yet, local recurrence has not been found to affect the survival as compared to amputation provided that it is treated adequately. ${ }^{12}$ Limb salvage surgery in sarcoma is aimed to balance between excision of tumor mass completely and leaving enough soft tissue for residual good function. The functional outcome after limb salvage surgery of STS is significantly better than amputation in many studies. $^{13}$

Extremity is the most common site of STS in our study overall which is consistent with the findings of Murrey et al. ${ }^{14}$ Lower extremity is the most common site of the STS in our study $(56.3 \%)$ which is in par with the other study. ${ }^{14,15}$

There is male predominance with male to female ratio of 7:1 in the current study. Hui et all have shown male predilection of STS in the ratio of 1.4:1 in their study. ${ }^{15}$ There is variability in the median age group as shown in different studies. Median age of 53 years was present in the study of Jurgen et al. ${ }^{16}$ Mean age of 32.88 years shown in the current study is different from the findings of mean of 61 years in the study of Kask et al. ${ }^{11}$ This variability could be due to small sample size of the present study.

Present study showed liposarcoma as the most common type of tumor (37.5\%) followed by rhabdomyosarcoma (25\%). There is wide variation in tissue types of STS in different studies. Murray et al in his study of 10,000 STS found liposarcoma as the most common types $(20 \%)$ followed by leomyosarcoma and undifferentiated $14 \%$ each. $^{14}$

The mean size of the STS in the present study 
was $19.5 \mathrm{~cm}$ (range 6-30) as measured on the side of maximum length. Soft tissue tumor $>5 \mathrm{~cm}$, rapidly increasing and deeper to the fascia are likely to be STS and must be investigated and ruled out accordingly. Similar study done by Murrey et al have shown $38 \%$ of the STS having size $>10 \mathrm{~cm}^{14}$

Most of the STS were stage I (50\%) followed by stage III (37.5\%) and stage II $(12.5 \%)$ in the current study. Grading of the tumor was found to be $62.5 \%, 18.8 \%$ and $18.8 \%$ for grade 1, 2 and 3 respectively. Grading has got significant correlation with final functional results ( $p=0.004$ ) with higher grade STS having lower MSTS score in the present study. This findings is consistent with findings shown by Eunsun et al. ${ }^{17}$ The important parameters that correlate with the final functional outcome are tumor size, age, grade of tumor, margin status, postoperative radiotherapy and chemotherapy. Present study showed weak positive correlation (correlation coefficient $=0.541$ ) between age and final MSTS score ( $p=0.03$ ) which is different to the findings shown by Eunsun et al. ${ }^{17}$ This could be explained by small sample size and more number of rhabdomyosarcoma in pediatric age group which gave rise to poor results.

Present study did not show significant correlation between tumor size and final functional score in contrary to the most of the studies. ${ }^{14,16,17}$ This could be because of less number of cases and presence of predominantly liposarcoma grade I tumor which have got good result after treatment in the current study. There is no significant correlation between sex and functional outcome in the present study. Present study showed significant correlation between postoperative RT and functional score $(\mathrm{p}<0.05)$. Similar positive correlation was present in the study done by Pister et al and Yang et al. ${ }^{18,} 19$ Many other studies have shown improved outcome with postoperative RT in the patients with close margins. ${ }^{9}$ Radiotherapy is beneficial and advised in all cases of high grade tumor and/or positive margin after excision.

There was also significant correlation between postoperative chemotherapy and functional score $\quad(p=0.007)$. Sarcoma Meta-Analysis Collaboration meta-analysis reported increased disease free survival with adjuvant CT after STS surgery but there is no overall increased survival. However, Present study could not analyze long term survival, local recurrence and metastasis because of lack of long term follow up and small sample size.

Present study showed mean MSTS functional score at the last follow up as 24.56 (81.83\%) with range from $19-29$ (63.33\%-96.66\%). There were good to excellent results in 13 cases ( $81.25 \%$ ) which are similar to the findings of Gerrand et al (mean MSTS 87.9\%) and Saebye et al (mean MSTS 92\%). ${ }^{20,21}$

However, present study has limitations due to less number of cases, short duration of follow up and retrospective nature of the study. This study did not analyze data regarding survivorship, local recurrence and metastasis as these analysis need long term follow up with large sample of data. Study involving larger number of cases or multicentric study with longer duration of follow up is advised in our country to validate the result of this study further.

\section{CONCLUSIONS}

Soft tissue sarcomas are uncommon variety of malignancy arising from different types of mesenchymal tissue. Limb salvage surgery of STS is the mainstay of treatment with or without supplementation with radiotherapy and chemotherapy. Current study provided preliminary demographic data of STS and mean MSTS functional score was found to be 24.56 $(81.83 \%)$. Good to excellent result was found in $81.25 \%$ of the cases as evaluated by MSTS score 
at minimum of 2 years of follow up. Positive correlation was found between radiotherapy, chemotherapy and age with final functional score and statistically significant negative correlation was found with grade of tumor. Further study with large number of cases with long term follow up is advised to validate the result of this study further.

\section{Conflict of Interest}

There is no conflict of interest to declare.

\section{REFERENCES}

1. R. Siegel DN, and A. Jemal. Cancer statistics, 2013. CA: A Cancer Journal for Clinicians,. 2013;63(No 1):pp. 11-30.

2. Kasper B GT, D'Hondt V, Gebhart M, Awada A. Novel treatment strategies for soft tissue sarcoma. Crit Rev Oncol Hematol. 2007;62:9-15.

3. Lewis VO. What's new in musculoskeletal oncology. Journal of Bone and Joint Surgery American volume. 2009.

4. R. Veth RvH, M. Pruszczynski, J. Hoogenhout, B.Schreuder, and T.Wobbes. , “Limb salvage in musculoskeletal oncology," Lancet Oncology. 2003; vol. 4(no. 6):343-50.

5. Bell RS OSB, Liu FF, et al. The surgical margin in soft-tissue sarcoma. J Bone Joint Surg. 1989;71A:370 \pm 5 .

6. Brennan MF CE, Harrison LB, et al. The role of multimodality therapy in softtissue sarcoma. Ann Surg 1991; 214; $328 \pm 36$. .

7. Brennan MF HB, Shiu MH, et al. Local recurrence in adult soft-tissue sarcoma. Arch Surg 1987; 122; 1289 \pm 93 .

8. Grimer RJI, PeakeD, Seddon B. Guidelines for the management of soft tissue sarcomas. Sarcoma. 2010;2010:506182.

9. Cakır S DF, Uzel O, Koca S, Okkan S.
Multivariate analysis of prognostic factors in 75 patients with soft tissue sarcoma. Radiother Oncol. 1995;37(1):10-16.

10. DSS. Introduction to limb-salvage surgery for sarcomas. Orthop Clin North Am 1991.

11. Kask G B-RI, Repo JP, Kjäldman M, Kilk K, Blomqvist C, Tukiainen EJ. Functional Outcome Measurement in Patients with Lower-Extremity Soft Tissue Sarcoma: A Systematic Literature Review.Ann Surg Oncol. 2019 Dec;26(13):4707-4722.

12. Rosenberg SA TJ, Glatstein E, et al. The treatment of soft-tissue sarcomas of the extremities: prospective randomized evaluations of (1) limb-sparing surgery plus radiation therapy compared with amputation and (2) the role of adjuvant chemotherapy. Ann Surg 1982;196:305e15.

13. Wright E GS, Gibbons C, Critchley P, Giele H. Functional and oncological outcomes after limb-salvage surgery for primary sarcomas of the upper limb. J Plast Reconstr Aesthet Surg 2008.

14. Brennan MF AC, Moraco N, et al. Lessons learned from the study of 10,000 patients with soft tissue sarcoma. Ann Surg 2014;260(3):416-22.

15. JY. H. Epidemiology and etiology of sarcomas. Surg Clin North Am. 2016;62:915.

16. Jürgen Weitz CRA aMFB. Localized 
Extremity Soft Tissue Sarcoma: Improved Knowledge With Unchanged Survival Over Time. Journal of Clinical Oncology. 2003.

17. Eunsun Oh, 2 Sung Wook Seo ", Han3 aKJ. A Longitudinal Study of Functional Outcomes in Patients with Limb Salvage Surgery for Soft Tissue Sarcoma. Sarcoma 2018.

18. Pisters PWT HL, Leung DH. Long-term results of a prospective randomized trial of adjuvant brachytherapy on soft tissue sarcoma. J Clin Oncol 1996;14:859-68.

19. Yang JC CA, Baker AR. Randomized prospective study of the benefit of adjuvant radiation therapy in the treatment of soft tissue sarcomas of the extremity. J Clin Oncol 1998;16:197-203.

20. Gerrand $\mathrm{CH}$ WJ, Kandel RA, et al. The influence of anatomic location on functional outcome in lower-extremity soft tissue sarcoma. Ann Surg Oncol. 2004;11:476-482.

21. Saebye C FH, Nymark T, et al. Factors associated with reduced functional outcome and quality of life in patients having limb-sparing surgery for soft tissue sarcomas: a national multicenter study of 128 patients. Acta Oncol. 2017;56:239-244.

Citation: Pandey S. Demography and Short Term Functional Outcome of Soft Tissue Sarcoma Treated with Limb Salvage Surgery. JCMS Nepal. 2020; 16(4):238-45. 